\title{
Spatial dynamics of homochiralization
}

\author{
Tuomas Multamäki and Axel Brandenburg \\ NORDITA, Blegdamsvej 17, DK-2100 Copenhagen Ø, Denmark
}

Received March 15, 2005; accepted May 4, 2005

\begin{abstract}
The emergence and spreading of chirality on the early Earth is considered by studying a set of reactiondiffusion equations based on a polymerization model. It is found that effective mixing of the early oceans is necessary to reach the present homochiral state. The possibility of introducing mass extinctions and modifying the emergence rate of life is discussed. Key Words: Homochirality - Origin of life - Exobiology.
\end{abstract}

\section{Introduction}

The question of the origin of life is closely connected with the question of how handedness (chirality) of living organisms came about (Avetisov et al. 1991). The sugars produced in plants via photosynthesis, for example via the rather innocently looking reaction

$$
6 \mathrm{CO}_{2}+6 \mathrm{H}_{2} \mathrm{O} \rightarrow \mathrm{C}_{6} \mathrm{H}_{12} \mathrm{O}_{6}+6 \mathrm{O}_{2},
$$

are all right-handed and rotate the polarization plane of polarized light in a right-handed sense, i.e. the sugar is said to be dextrorotatory. This preferred handedness is remarkable because the difference in binding energy between right-handed and lefthanded (levorotatory) sugars is extremely tiny (relative energy difference: $10^{-17}$ ). Once dead, the preferred handedness gradually disappears until there are equally many right and left handed molecules. This process, which speeds up with increasing temperature, is called racemization and can even be used as an approximate dating method (Hare \& Mitterer 1967, Bada et al. 1970). For a simpler and closely related reaction that displays a similar behavior we refer to the formose reaction discussed by Toxvaerd (2005).

Another type of dextrorotatory sugar with five carbon atoms occurs in the backbone of RNA, for example, making RNA therefore chiral. RNA can have autocatalytic properties, which led to the idea of an early "RNA world" (Gilbert 1986, Joyce 1991) where naked RNA molecules could have catalyzed the polymerization of other RNA molecules. But since the RNA molecule consists of right-handed sugars, chirality selection must have occurred at an even earlier stage.

Two possibilities are commonly discussed. Either chirality was imposed by physical factors such as magnetic fields, polarized light from a nearby pulsar, or even the tiny energetic preference due to the parity-breaking electroweak interaction. In such a scenario, homochirality may well have been a prerequisite for the origin of life, allowing for example for the assembly of structurally more stable polymers. The difficulty here is that such mechanisms would hardly explain the complete homochirality observed in living matter. The other possibility is that homochirality was a consequence of life, and that the presently prevailing handedness was selected spontaneously during the assembly of the first polymers. As shown in the early paper by Frank (1953), such a process requires the production of left and right handed molecules to be mutually antagonistic. He devised pairs of ordinary differential equations demonstrating how this could possibly be described mathematically.

The latter scenario is the one we consider also in the present paper. Significant progress has been made since Frank's early paper. In particular, the 
origin of the mutually antagonistic behavior has been identified to be the so-called enantiomeric crossinhibition, a process that spoils further polymerization once a monomer of opposite handedness has been attached to an already existing polymer. The evidence for the occurrence of this process is entirely experimental and goes back to the early work of Joyce et al. (1984), who found that in non-enzymatic template-directed polymerization of RNA strands, only a homochiral supply of mononucleotides that are complementary to the template can polymerize to a typical length of 20 nucleotides. Even a small amount of mononucleotides of opposite chirality prevents the formation of longer polymers, as is seen in high performance color chromatograms. Similar experiments have subsequently also been carried out by Schmidt et al. (1997) and Kozlov et al. (1998), for example. In the following we describe in more detail how this process can be modeled.

\section{The polymerization model}

In the polymerization model of Sandars (2003) large polymers are generated by joining successive monomers into long chains. Monomers can be both left, $L_{1}$, and right, $R_{1}$, handed, and longer chains are formed according to the following set of reaction equations:

$$
\begin{array}{rll}
L_{n}+L_{1} & \stackrel{2 k_{S}}{\longrightarrow} & L_{n+1}, \\
L_{n}+R_{1} & \stackrel{2 k_{I}}{\longrightarrow} L_{n} R_{1}, \\
L_{1}+L_{n} R_{1} & \stackrel{k_{S}}{\longrightarrow} & L_{n+1} R_{1}, \\
R_{1}+L_{n} R_{1} & \stackrel{k_{I}}{\longrightarrow} & R_{1} L_{n} R_{1},
\end{array}
$$

where $k_{S}$ and $k_{I}$ are the reaction coefficients for monomers to a polymer of the same or of the opposite handedness, respectively. For all four equations we also have the complementary reactions obtained by exchanging $L \rightleftharpoons R$. Note that chains are 'spoiled' if a monomer of opposite chirality is attached to the end of a longer chain, in which case the chain can only grow at the other end. This is in essence what is meant by enantiomeric cross-inhibition. As a results, polymers such as $R_{1} L_{2} R_{1}$ and $L_{1} R_{1}$ can no longer grow. As discussed in the introduction, this rule is motivated by the experiments of Joyce et al. (1984) and others.

The monomers are initially generated from a substrate $S$ according to

$$
S^{\stackrel{k_{C} C_{R}}{\longrightarrow}} R_{1}, \quad S^{\stackrel{k_{C} C_{\zeta}}{\longrightarrow}} L_{1}
$$

where $k_{C}$ is proportional to the regeneration rate of monomers, and $C_{R}$ and $C_{L}$ determine the enzymatic enhancement of right and left handed monomers. The dependence of $C_{L, R}$ on the existing amount of polymer chains is essential to chirality selection as then an existing excess of either chirality can be amplified. The exact form of $C$ is not crucial and here we follow the choice $C_{A}=\sum n A_{n}, A=L, R$ (Brandenburg et al. 2005, hereafter referred to as BAHN). For alternative prescriptions of $C_{A}$ we refer to the papers by Sandars (2003) and Wattis \& Coveney (2005). The substrate itself is being replenished by a constant source term $Q$.

The polymerization process is represented pictorially in Fig. 1, where one can see how monomers begin to grow into longer chains and can then be contaminated by a monomer of opposing chirality. The crossed out chains represent polymers that can no longer grow.

The feedback mechanism built into the polymerization model leads to an unstable system that, depending on the fidelity $f$ of the enzymatic reactions, when perturbed from the initial racemic state can reach a homochiral state. The prescription for $C_{A}$ discussed above assumes $f=1$. If $f<1$, there is some 'crosstalk' between $L$ and $R$ where $f$ determines the relative mixing between the two chiralities.

The chirality of a particular state is conveniently parameterized by a parameter called the enantiomeric excess:

$$
\eta \equiv \frac{E_{R}-E_{L}}{E_{R}+E_{L}},
$$

where $E_{A}=\sum n A_{n}$ with $A=L, R$. For $f$ larger than a critical value (see BAHN for details), the racemic state $(\eta=0)$ is unstable with respect to small perturbations. Only for $f=1$, the end state is fully homochiral $(\eta= \pm 1)$. 


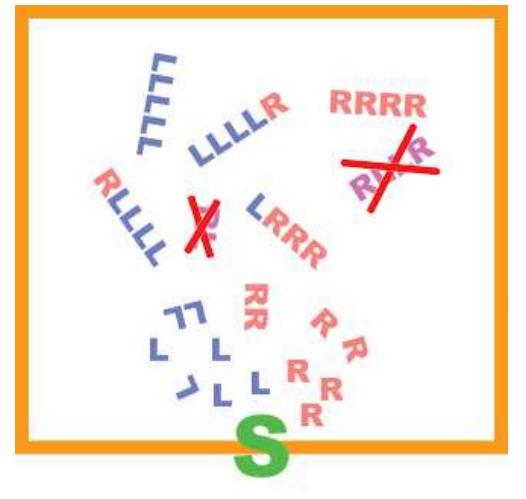

Figure 1: Polymerization process. In addition to a number of monomers, $\mathrm{L}$ and $\mathrm{R}$, there are several isotactic dimers, LL and RR, as well as longer polymers. Semi-spoiled polymers such as LLLLR and LRRR can still polymerize on the unspoiled end. Polymers such as LR and RLLR are dead and cannot polymerize further.

\section{Homochiralization in space}

The homochiralization process considered here and, indeed, in much of the theoretical literature on the subject (e.g., Frank 1953, Wei-Min 1982, Goldanskii and Kuzmin 1989, Avetisov \& Goldanskii 1993, Sandars 2003, Saito and Hyuga 2004a) has been uniform in space. Relaxing this restriction can, in principle, lead to completely new chirality selection mechanisms that have no analogue in models without spatial extend. An example is the mechanism identified by Toxvaerd (2004) using molecular dynamics simulations. In the present work we simply extend the homochiralization process discussed in the previous section to allow for the interaction with neighboring regions through diffusion and/or advection (such as ocean currents). We begin with a state that is homochiral everywhere, but that there are minute imbalances in space, i.e. infinitesimally small perturbations. These perturbations grow locally, leading to patches with enantiomeric excess of either handedness. It is then natural to ask how a localized homochiral state spreads to a racemic surroundings and, moreover, can regions of different chirality coexist?

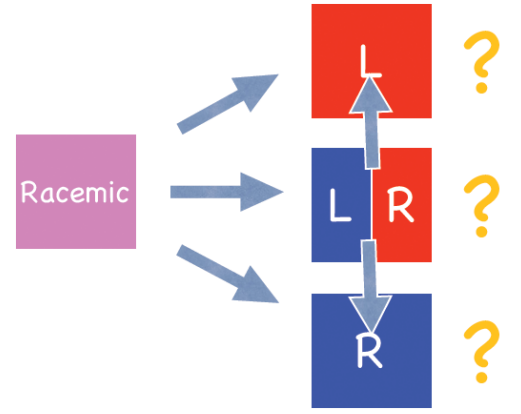

Figure 2: Different ways of reaching a homochiral state from an initial racemic state

This question is not restricted to the model considered here and needs to be addressed in all models where chirality is selected locally by a random process.

The critical ingredients necessary to answer these questions are the presence of different relevant time scales. If the appearance of a homochiral region is a rare process, say with a period of $\tau_{\text {life }}$, and the global homochiralization time scale, $\tau_{\text {global }}$, is short compared to that, i.e. $\tau_{\text {global }} \ll \tau_{\text {life }}$, then one would not expect coexisting regions of different chirality. In other words, if the emergence of life is a rare event, then life forms with different handedness did not probably coexist. This is the case modeled by Frank (1953) and many others after him. In the other extreme case, homochiral regions appear frequently compared to the speed at which they can dominate the early Earth, leading to coexisting regions of opposite chirality (see Fig. 2). Even though here we are mainly concerned with chirality, such arguments can be applied more generally to the emergence and spreading of life as well. For a discussion of the possibility of finding a second sample of life on Earth see the recent paper by Davies \& Lineweaver (2005).

The spreading of chirality and coexistence of regions of different chirality was studied in Brandenburg and Multamäki (2004), hereafter referred to as BM. There a reduced polymerization model with spatial extent was considered analytically and by numerical simulations. The importance of spatial extent has already been emphasized by Saito and 
Hyuga (2004b) who generalized the model of Saito and Hyuga (2004a) by using a Monte-Carlo method as it is used in percolation studies.

The behavior of the reduced model can be understood qualitatively: an initial racemic mixture with small local perturbations quickly relaxes locally to regions of different chirality. Hence, in this work the emergence of life was considered to be a frequent and rapid event. The oppositely handed regions then begin to spread spatially into any possibly remaining racemic regions by front propagation until they come in contact with a region of opposite chirality. In $1+1$ dimensions (one space and one time dimension) there is no further evolution and one can view the process as spontaneous symmetry breaking leading to stable, non-propagating domain walls. In more than one spatial dimension, the homochiralization process progresses further. If no advection is present, only diffusion can drive the homochiralization process. Analytical arguments can be utilized to show that a bubble surrounded by a region of opposite chirality tends to shrink. Since the equations are local, the local curvature of the front is the deciding property in determining which way a front will move. An example of this process is shown in Fig. 3, where we show a $2+1$ dimensional box with regions of different chirality. The initial state in the simulation was a racemic state with small fluctuations so that one quickly arrives at a state where there are many left and right handed regions in the box. Here we show the evolution of these homochiral regions. From the figure it is easy to see that the curvature of the interface indeed determines the evolution of a region: e.g. small bubbles shrink and later disappear.

Again, time scales play a crucial role in the global homochiralization process. Using microphysically motivated values, we find that the local relaxation to a homochiral state is rapid compared to that of the propagation of fronts into racemic surroundings. In turn, this is a much faster process than the final stage in global homochiralization when the regions of different chirality slowly disappear as described above. Using values of molecular diffusion, one actually finds that this time scale on Earth is much too long to explain the observed homochiral final state.

In order to study the process further, we have also
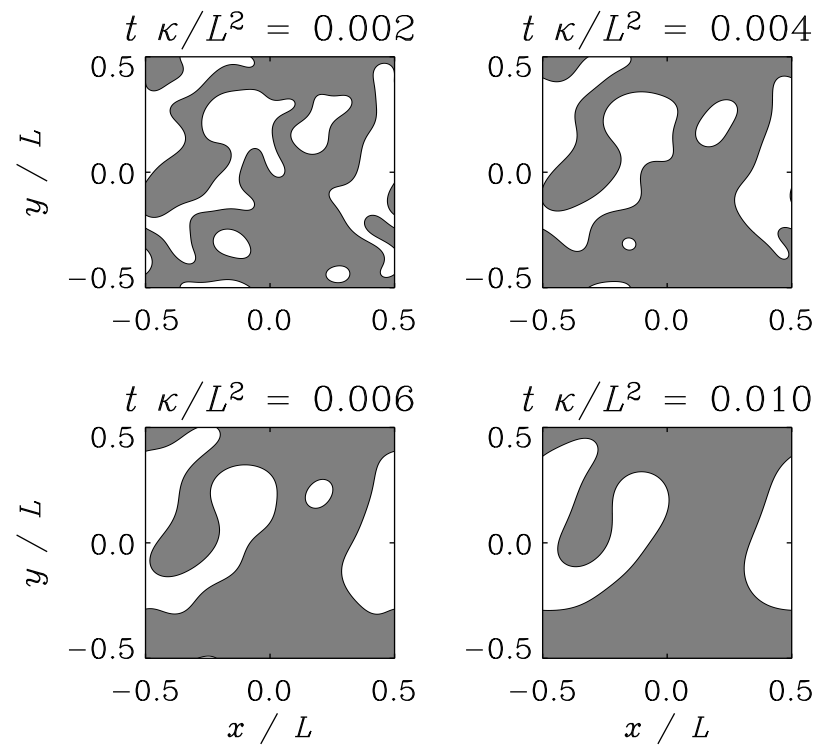

Figure 3: The spreading of homochiral regions in $2+1$ dimensions. The dark (light) regions correspond to left (right) handed regions. Time is given in global diffusion times, $\tau_{\text {turb }}=L^{2} / \kappa$. (Adapted from BM.)

considered the effects of advection on homochiralization. An example in $2+1$ dimensions is shown in Fig. 4. The mixing process is now greatly enhanced and one finds that the global homochiral state is reached much more rapidly. The actual time scale depends strongly on the strength of the flow, e.g. for root mean square flow of $1 \mathrm{~cm} / \mathrm{s}$, the time scale of global homochiralization is of the order of 30 years. It is then clear that if the model considered in BM captures the relevant features of chirality selection on the early Earth, the effective mixing of early oceans is vital. As the mixing is affected by many factors such as the existence of continents and salinity of sea water, it is not difficult to speculate that in some secluded parts of the early oceans, life forms of different chirality could have coexisted.

So far we have considered the spreading in two horizontal directions, which is relevant to the Earth's surface where the vertical dynamics may be eliminated by vertical averaging. However, BM also considered three-dimensional models and found that the speed of 

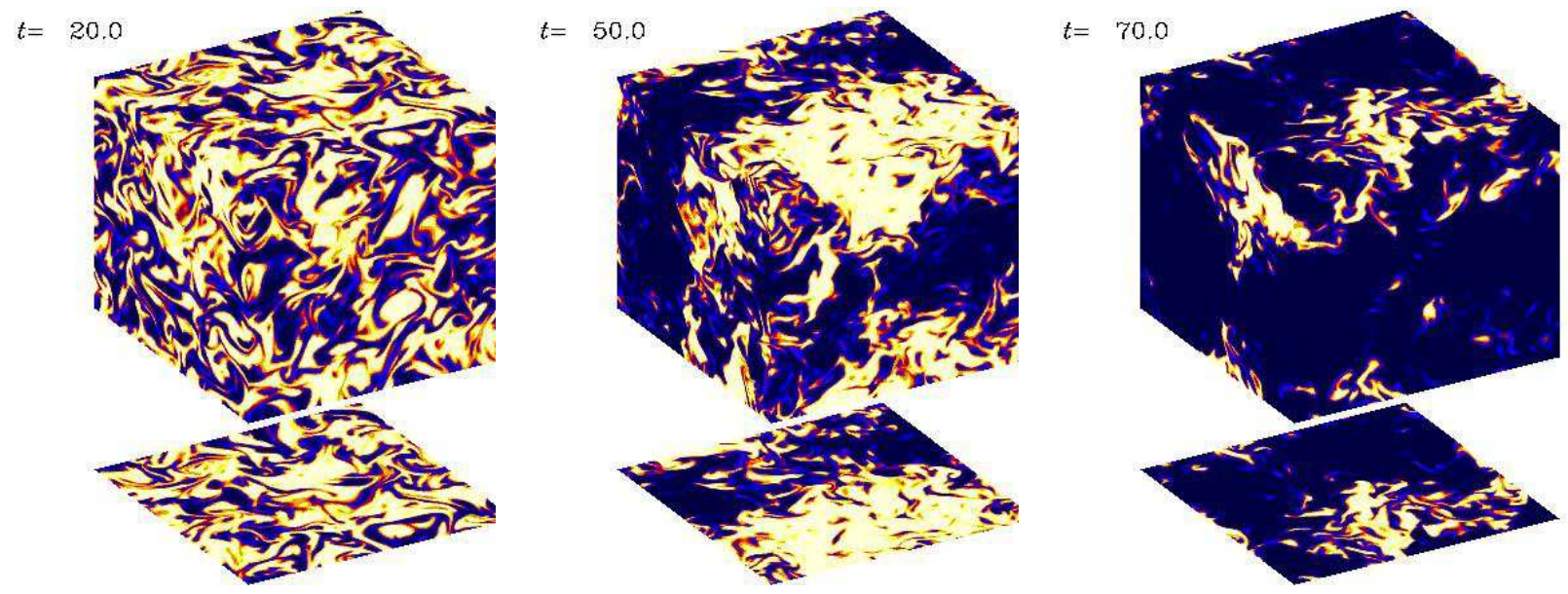

Figure 5: The spreading of homochiral regions in $3+1$ dimensions. The dark (light) regions correspond to left (right) handed regions. The time $t=50$ corresponds here to $t / \tau_{\text {turb }} \approx 2$. This is also the last time shown in Fig. 4 . By the time $t=100$ corresponds here to $t / \tau_{\text {turb }} \approx 4$, the right handed life form went extinct.
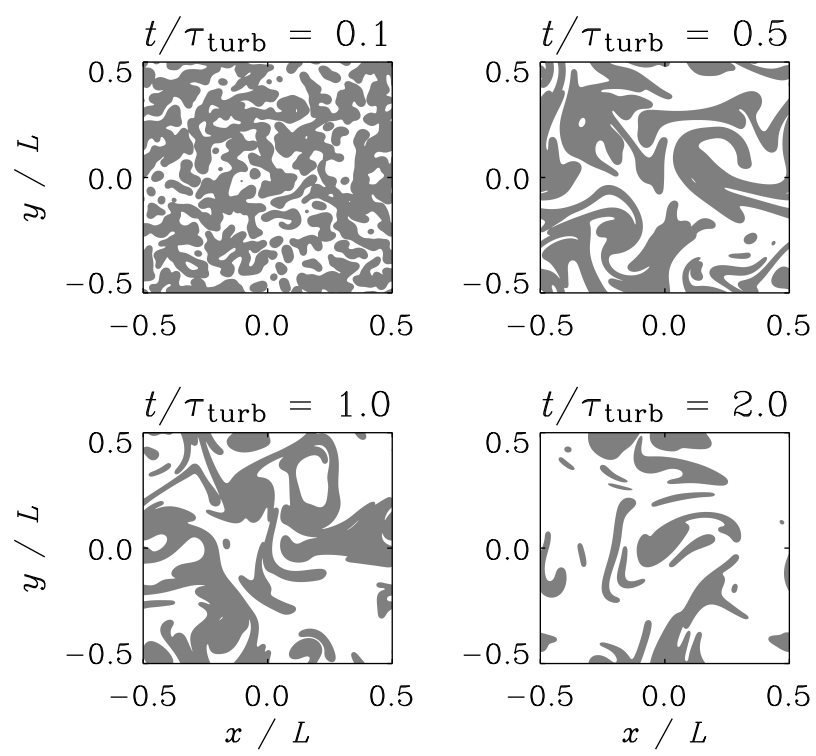

Figure 4: The spreading of homochiral regions in $2+1$ dimensions. The dark (light) regions correspond to left (right) handed regions. Time is given in dynamical times, $\tau_{\text {turb }}=\ell / u_{\text {rms. }}$. (Adapted from BM.) homochiralization is somewhat enhanced (by about $30 \%)$ relative to the two-dimensional case. Furthermore, in both two and three dimensions they found that the generalized enantiomeric excess,

$$
\eta=\frac{\left\langle E_{R}\right\rangle-\left\langle E_{L}\right\rangle}{\left\langle E_{R}\right\rangle+\left\langle E_{L}\right\rangle}
$$

where angular brackets denote averages in space, grows approximately linearly in time:

$$
\frac{\mathrm{d} \eta}{\mathrm{d} t}=(12 \ldots 18) \times \mathcal{N}\left(\frac{2 \lambda^{2}}{Q k_{S}}\right)^{1 / 4} \frac{\kappa}{L^{2}},
$$

where $\mathcal{N}$ is the number of topologically disconnected domains, $\lambda / \sqrt{Q k_{S}}$ is the non-dimensional growth rate in the spatially uniform case, $L$ is the spatial extent of the domain, and $\kappa$ is the diffusion coefficient.

In the turbulent case, $\kappa$ can simply be replaced by a turbulent coefficient $\kappa_{\text {turb }}$, for which BM found from their simulation the rough estimate $\kappa_{\text {turb }} \approx$ $(0.08 \ldots 0.15) u_{\mathrm{rms}} \ell$, where $u_{\mathrm{rms}}$ is the root mean square velocity of the turbulence and $\ell$ is the typical length scale of the turbulent eddies. It should be emphasized, however, that such a description of turbulent 
mixing is rather rudimentary and ignores, for example, the fact that the shape of left and right handed domains is quite different with and without turbulence: it is more round in the case of molecular diffusion and more elongated in the turbulent case. The latter is a feature of the turbulence to mix fields by stretching and folding. A snapshot of a fully threedimensional simulation ${ }^{1}$ showing the concentration of left handed building blocks is presented in Fig. 5 .

\section{Emergence and spreading of life on the early Earth}

We have made a number of underlying assumptions in studying the spreading of homochiral domains on the early Earth. Firstly, we have assumed that the initial state is globally racemic. This implies that the conditions were life could have emerged are uniform and favorable everywhere and, furthermore, that a homochiral region can spread and fill the whole domain. Secondly, we have assumed that the emergence of life is a common event and that it is possible everywhere on earth, and that there are no mass extinctions. These assumptions may not necessarily be realistic, and one should consider what new effects can possibly arise from relaxing these assumptions.

Considering the possibility of mass extinctions and that the emergence of life can be a rare event, it is clear that a new time scale, $\tau_{\text {ext }}$, is inserted into the process. Assuming that mass extinctions are local events (i.e. not all life is destroyed in which case the process can start again), the relative lengths of the different time scales determine the qualitative dynamics of the system. If $\tau_{\text {global }} \ll \tau_{\text {life }} \ll \tau_{\text {ext }}$, life appears somewhere and takes over the whole system before life of opposite chirality can emerge. Any later mass extinction events will be insignificant as the dominant chirality will quickly win over the achiral region. If $\tau_{\text {life }} \ll \tau_{\text {global }} \ll \tau_{\text {ext }}$, we have the situation considered earlier in this paper, where regions of different chirality compete. If mass extinctions are

\footnotetext{
${ }^{1}$ Animations of both turbulent and purely diffusive solutions can be found at http://www.nordita.dk/ brandenb/ movies/chiral
}

common, $\tau_{\text {ext }} \ll\left(\tau_{\text {life }}, \tau_{\text {global }}\right)$, and so any emergent life will quickly be wiped out - at least locally. Again, if $\tau_{\text {global }} \ll \tau_{\text {life }}$, it is unlikely that life will re-emerge spontaneously in the affected areas, and these areas will more likely be re-populated by the spreading of the homochiral regions surrounding the now racemic area. Thus, in this case homochirality is preserved. On the other hand, if $\tau_{\text {life }} \ll \tau_{\text {global }}$, the possibility of mass extinctions allows for new life forms to emerge which, in turn, may prolong the time during which life forms of opposite chirality can have coexisted.

It is not immediately obvious how mass extinctions should be modeled within the framework of the BAHN model. It seems plausible that a mass extinction due to an impact, for example, can be modeled by removing all existing polymers. The re-emergence of life depends on the source term $Q$ of the substrate and on the $k_{C}$ parameter that controls the rate at which new monomers are created from the substrate. By locally suppressing $Q$ or $k_{C}$ as a consequence of the mass extinction, one can model the reduced habitability by slowing down the generation either of the substrate or of new monomers from the source. Alternatively, one could decrease the polymerization efficiency by decreasing $k_{S}$ and $k_{I}$ on the grounds that these parameters are likely to depend on external factors such as temperature, salinity, and acidity.

Allowing for the additional dependencies discussed above introduces certainly a lot of additional uncertainties, but it also removes some of the sensitivity to initial conditions. If the conditions for chirality (or life) are not satisfied everywhere, the spreading of chirality will stop and mass extinctions can eradicate the emergent life forms. Hence, there are various approaches to modeling the emergence of life and mass extinctions in the BAHN model. Either one can deplete the source of the substrate, or one can diminish the conversion rates for monomer formation and/or for polymerization, in which case the substrate might still be present everywhere.

Clearly, these considerations are relevant in a more realistic model and, furthermore, can bring interesting new dynamics into the system. As such they are worth considering in more detail and future work will hopefully bring us answers to these questions. Detailed numerical models of this process may elucidate 
further the possible outcomes. We plan to adapt the PENCIL CODE ${ }^{2}$, which was also used in our earlier paper, to include the effects of a dynamical evolution of the rate at which new left and right handed monomers can be regenerated.

\section{Conclusions}

We have studied the spatial evolution of chirality in an initially racemic system. By utilizing analytical and numerical methods, we have shown how a globally homochiral state can be reached. The associated time scale depends strongly on the details of the system, but molecular diffusion is too slow to homochiralize the early Earth and turbulent flows are necessary. Effective mixing of the early oceans drastically reduces the required time and is hence a vital ingredient in understanding the homochiralization process.

We also consider qualitatively how a more infrequent emergence of homochiral regions and the addition of mass extinctions would affect the evolution of the system. Adding such (possibly more realistic) processes enriches the dynamics and offers new interesting avenues of research to be explored in the future.

\section{Acknowledgments}

The Danish Center for Scientific Computing is acknowledged for granting time on the Linux cluster in Odense (Horseshoe).

\section{References}

Avetisov, V. A., Goldanskii, V. I., and Kuz'min, V. V. (1991) Handedness, origin of life and evolution, Phys. Today 44, 33-41.

Avetisov, V. A. and Goldanskii, V. (1993) Chirality and the equation of 'biological big bang', Phys. Lett. A 172, 407-410.

Bada, J. L., Luyendyk, B. P., and Maynard, J. B. (1970) Marine sediments: dating by the

\footnotetext{
${ }^{2}$ http://www.nordita.dk/software/pencil-code
}

racemization of amino acids, Science $\mathbf{1 7 0}$, 730-732.

Brandenburg, A., Andersen, A. C., Höfner, S., and Nilsson, M. (2005) Homochiral growth through enantiomeric cross-inhibition, Orig. Life Evol. Biosph. (in press). Preprints available online at: http://arXiv.org/abs/ q-bio/0401036 (BAHN).

Brandenburg, A. and Multamäki, T. (2004) How long can left and right handed life forms coexist?, Int. J. Astrobiol. 3, 209-219.

Davies, P. C. W. and Lineweaver, C. H. (2005) Finding a second sample of life on Earth, Astrobiol. 5, 154-163.

Frank, F. C. (1953) On Spontaneous Asymmetric Synthesis, Biochim. Biophys. Acta 11, 459464.

Gilbert, W. (1986) Origin of life - the RNA world, Nature 319, 618-618.

Goldanskii, V. I. and Kuzmin, V. V. (1989) Spontaneous breaking of mirror symmetry in nature and origin of life, Sov. Phys. Uspekhi 32, 1-29.

Hare, P. E. and Mitterer, R. M. (1967) Nonprotein amino acids in fossil shells, Yearbook Carnegie Institution of Washington 65, 362-364.

Joyce, G. F., Visser, G. M., van Boeckel, C. A. A., van Boom, J. H., Orgel, L. E., and Westrenen, J. (1984) Chiral selection in poly(C)-directed synthesis of oligo(G), Nature 310, 602-603.

Joyce, G. F. (1991) The rise and fall of the RNA world, New Biol. 3, 399-407.

Kozlov, I. A., Pitsch, S., and Orgel, L. E. (1998) Oligomerization of activated D- and Lguanosine mononucleotides on templates containing D- and L-deoxycytidylate residues, Proc. Natl. Acad. Sci. 95, 13448-13452.

Saito, Y. and Hyuga, H. (2004a) Complete homochirality induced by the nonlinear autocatalysis and recycling, J. Phys. Soc. Jap. 73, 33-35.

Saito, Y. and Hyuga, H. (2004b) Homochirality proliferation in space, J. Phys. Soc. Jap. 73, 1685-1688.

Sandars, P. G. H. (2003) A toy model for the generation of homochirality during polymerization, 
Orig. Life Evol. Biosph. 33, 575-587.

Schmidt, J. G., Nielsen, P. E., \& Orgel, L. E. (1997) Enantiomeric cross-inhibition in the synthesis of oligonucleotides on a nonchiral template, $J$. Am. Chem. Soc. 119, 1494-1495.

Toxvaerd, S. (2004) Domain catalyzed chemical reactions: a molecular dynamics simulation of isomerization kinetics, J. Chem. Phys. 120, 6094-6099.

Toxvaerd, S. (2005) Origin of homochirality in biological systems, Int. J. Astrobiol. 4 (in press).

Wattis, J. A. D. and Coveney, P. V.: 2004, Symmetry-breaking in chiral polymerisation, Orig. Life Evol. Biosph. (in press), arXiv:physics/0402091.

Wei-Min, L. (1982) Remarks on origins of biomolecular asymmetry, Orig. Life 12, 205-209.

Id: paper.tex,v 1.26 2005/05/21 06:01:25 brandenb

Exp 